\title{
Stability and performance analysis of an irrigation channel with distributed control*
}

\author{
Y. Li and B. De Schutter
}

If you want to cite this report, please use the following reference instead:

\begin{abstract}
Y. Li and B. De Schutter, "Stability and performance analysis of an irrigation channel with distributed control," Control Engineering Practice, vol. 19, no. 10, pp. 11471156, Oct. 2011.
\end{abstract}

Delft Center for Systems and Control Delft University of Technology

Mekelweg 2, 2628 CD Delft

The Netherlands

phone: +31-15-278.24.73 (secretary)

URL: https: //www.dcsc.tudelft.nl 


\title{
Stability and Performance Analysis of an Irrigation Channel with Distributed Control ${ }^{\text {th }}$
}

\author{
Yuping $\mathrm{Li}^{*}$, Bart De Schutter \\ Delft Center for Systems and Control, Delft University of Technology, Mekelweg 2, 2628 \\ $C D$, Delft, the Netherlands.
}

\begin{abstract}
For a string of pools with distant-downstream control, the internal time-delay for water transport from upstream to downstream does not only limit the local control performance of regulating water-levels at setpoints and rejecting offtake disturbances in each pool, but also impacts the global performance of managing the water-level error propagation and attenuating the amplification of control actions in the upstream direction. A distributed control scheme that inherits the interconnection structure of the plant is investigated. It is shown that the decoupling terms in the controller help to improve global closed-loop performance by decreasing the low-frequency gain of the closed-loop coupling. Moreover, the decoupling terms compensate for the influence of the time-delay by imposing extra phase lead-lag compensation in the mid-frequency range on the closed-loop coupling function.
\end{abstract}

Keywords: Distributed control, Feedforward compensation, Internal time-delay, Irrigation channels, Performance trade-off

\section{Introduction}

Water is becoming a scarce resource all over the world. Globally, irrigation now accounts for 69 percent of the 3240 cubic kilometers ( 772 cubic miles) of water withdrawn for human use, and 87 percent of all water consumed (Agri and Wat, 2010). The majority of irrigation is achieved through an extensive civil

\footnotetext{
in This work was supported in part by the European 7th framework STREP project "Hierarchical and distributed model predictive control of large-scale systems (HD-MPC)", contract number INFSO-ICT-223854, the Delft Research Center NGI, the BSIK project "Next Generation Infrastructures (NGI)", and the European 7th Framework Network of Excellence "Highly-complex and networked control systems (HYCON2)", and in part by the Australian Research Council (Linkage Grant LP0349134) when the first author was working with the University of Melbourne.

* Corresponding author.

Email addresses: yuping.li@tudelft.nl (Yuping Li), b.deschutter@tudelft.nl (Bart De Schutter)
} 
infrastructure of reservoirs and open channels that supplies fresh water to farms. Fig. 1 shows the topview of a typical irrigation network. Water is drawn from

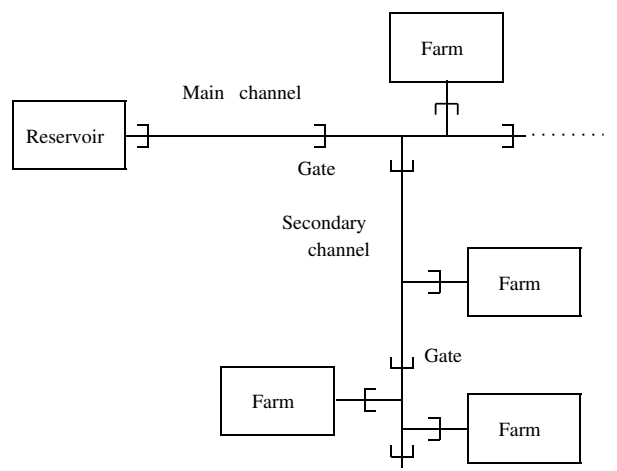

Figure 1: Topview of an irrigation network

the reservoir and distributed through the main channel and many secondary channels to farms. Along the channels, mechanical gates are installed to regulate the flow. A stretch of water between two neighboring gates is called a pool. An irrigation network is usually largely gravity-fed (i.e. there is no pumping); to satisfy water-demands from farms and to decrease water wastage, the waterlevels in the pools should be regulated to certain setpoints. Since most farms sit at the downstream ends of pools, it is more important to control downstream water-levels. To avoid excessive communication load for large-scale irrigation systems, decentralized control is preferred to centralized control. In practice, a distant-downstream control structure (i.e. use the upstream gate to control the downstream water-level of a pool) is implemented for good management of water service and water distribution efficiency (Litrico and Fromion, 2003). Such a control strategy combines the advantages of downstream control avoiding excess water losses at the tail end of the canal, and those of controlling a water-level upstream of cross devices allowing low water elevation in the canal pools. These low water elevations reduce the cost of civil engineering works since banks can follow the natural ground levels (Malaterre et al., 1998). Hence, the current paper focuses on distant-downstream control.

Further, an irrigation channel is a system presenting strong interactions between pools, i.e. the flow into a pool is equal to the flow out of the neighboring upstream pool. With distant-downstream control, when offtakes occur at a downstream pool, one could see amplification of the control actions (e.g. flows over upstream gates) and water-level error propagation in the upstream direction. ${ }^{1}$ The coupling effects between pools, and several ways of reducing them

\footnotetext{
${ }^{1}$ In instant-upstream control (i.e. using the downstream gate to control the downstream water-level of a pool), there exists water-level error propagation in the downstream direction, while in a general centralized control scheme, the closed-loop coupling is in both the upstream and the downstream direction.
} 
have already been studied in the past, see (Deltour, 1992; Malaterre and Baume, 1999; Schuurmans, 1992). In particular, (Schuurmans, 1992) proposed two decouplers to reduce the coupling effects between neighboring SISO subsystems. In (Deltour, 1992), a "PIR" controller is suggested, which, as pointed out in (Malaterre, 1994), has two features that were indicated to be equivalent to the two decouplers of (Schuurmans, 1992). The analysis in (Malaterre and Baume, 1999) show that when distant-downstream control structure is chosen, the above decoupling measures are actually a feedforward compensation. Indeed, control objectives for large-scale irrigation networks involve: locally, setpoints regulation, rejection of offtake disturbances, avoiding excitement of dominant waves and, globally, management of the water-level error propagation and attenuation of the amplification of control actions in the upstream direction. As shown in (Li et al., 2005), there exists a trade-off between the local and the global control performance. To cope with the trade-off between the local and the global control performance, a distributed control scheme that inherits the interconnecting structure of the plant is suggested in (Cantoni et al., 2007; Li and Cantoni, 2008). The distributed distant-downstream controller is designed through the formulation of a structured optimal control problem, based on loop-shaping ideas. The performance index in the cost function captures the trade-off between the local performance and the global performance. Field test results (see Section 2.4) show that such a distributed control scheme presents a performance advantage over the decentralized feedback with feedforward control scheme proposed in (Malaterre and Baume, 1999). In fact, as discussed in Section 2.3, decentralized feedback with feedforward control is a special realization of the distributed control scheme. However, systematically exploiting the feedforward compensators to manage the interactions between pools is difficult ( $\mathrm{Li}$ and Cantoni, 2008).

In this paper, to obtain a better performance and a simpler realization of the distributed controller, some changes are made in the controller configuration and the structure of the shaping weights of the distributed controller from those suggested in (Li and Cantoni, 2008), as discussed in Section 2.3. Different from (Cantoni et al., 2007; Li and Cantoni, 2008), which mainly discusses controller design, this paper focuses on an in-depth performance analysis of the distributed control scheme; more precisely, based on an analysis of the frequency response of closed-loop transfer functions, we investigate how the decoupling terms in the distributed distant-downstream controller help to attenuate the interactions between pools.

One critical issue in control design for an irrigation network comes from the time-delay in each pool, i.e. the time for transporting water from the upstream gate to the downstream gate. In this paper, the impacts of the internal timedelays on the local and global control performance are analyzed. Moreover, a discussion is included on how the distributed control scheme compensates for such impacts. Note that the influence of such an internal time-delay on the closed-loop performance with decentralized feedback and feedforward control has been analyzed in (Welz et al., 2005), in which the authors suggested to use a lead-lag filter approximating the internal time-delay over a given frequency as the feedforward compensator. However, how to set up such a lead-lag filter is 
not clear. Indeed, in the example given in (Welz et al., 2005), a static feedforward compensator is applied instead. In Section 4.2.3, analysis shows that the decoupling terms in the distributed controller operate on the closed-loop coupling function by imposing an additional phase lead-lag compensation around the frequency of the internal time-delay. This, to some extent, confirms the idea of feedforward compensator design given in (Welz et al., 2005). Moreover, the corresponding decoupling terms of the distributed controller give an example of how to design the lead-lag filter of the feedforward compensator.

The paper is organized as follows. Section 2 briefly introduces modeling of an irrigation channel and designing of the distributed controller to manage the trade-off between local and global performance. Field test results are given which demonstrate the performance of the distributed control scheme. The impact of the internal time-delay on the closed-loop performance is discussed in Section 3. Further, in Section 4 an analysis is performed on how the decoupling terms in the distributed controller help in managing the water-level error propagation and attenuating the amplification of control actions in the upstream direction. Section 5 concludes the paper.

\section{Plant model and distributed controller design}

This section serves to summarize the distributed controller design framework introduced in ( $\mathrm{Li}$ and Cantoni, 2008), which is the basis of the performance analysis in Sections 3 and 4.

\subsection{Plant model}

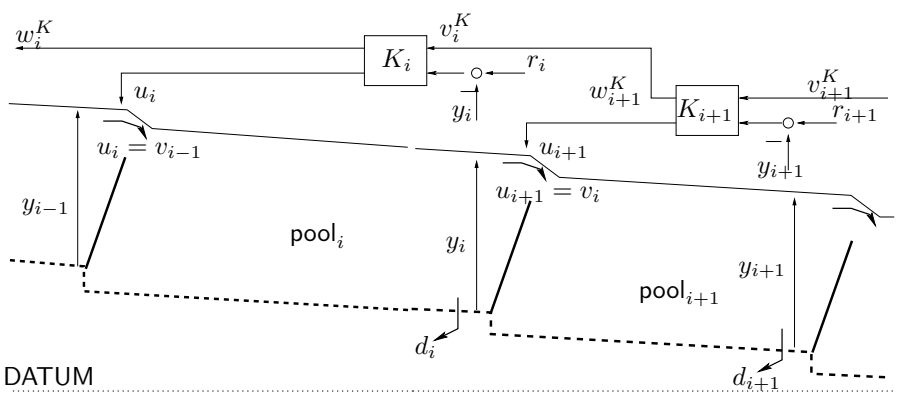

Figure 2: Distributed control of an open water channel

An irrigation channel is in fact a string of pools, see Fig. 2 for the sideview of a channel. Within the context of control design for setpoint regulation and load disturbance rejection, the following model, which is based in principle on conservation of mass, is employed:

$$
\alpha_{i} \dot{y}_{i}(t)=u_{i}\left(t-\tau_{i}\right)-v_{i}(t)-d_{i}(t),
$$


where $y_{i}(t)$ is the downstream water-level of $\operatorname{pool}_{i}, u_{i}(t)$ is the flow over the upstream gate, $v_{i}(t)$ the flow over the downstream gate, $d_{i}(t)$ models the offtake load-disturbances from pool $_{i}, \tau_{i}$ is the transport delay of water from the upstream gate to the downstream gate of the pool, and $\alpha_{i}$ is a measure of the pool surface area. Note the interconnection

$$
v_{i}(t)=u_{i+1}(t)
$$

i.e. the flow out from pool $_{i}$ equals the flow into pool $i+1$. As pointed out in Cantoni et al. (2007), such a first-order model is adequate for control design because it captures the dynamics of the water-levels from the perspective of the required closed-loop behavior. A model of this kind was used for feedback controller design in (e.g., Cantoni et al., 2007; Li and Cantoni, 2008; Li and De Schutter, 2010; Litrico and Fromion, 2005; Mareels et al., 2005; Overloop et al., 2005; Schuurmans et al., 1999; Clemmens and Schuurmans, 2004; Weyer, 2001). Taking the Laplace transform of (1), yields

$$
P_{i}: y_{i}(s)=\frac{1}{s \alpha_{i}}\left(e^{-s \tau_{i}} u_{i}-v_{i}-d_{i}\right)(s)
$$

\subsection{Trade-off between local performance and global performance}

Denote $e_{i}:=r_{i}-y_{i}$ the water-level error in $\operatorname{pool}_{i}$, with $r_{i}$ the setpoint of $y_{i}{ }^{2}$ Local control performance requires $e_{i}$ to be small in the presence of $d_{i}$; while closed-loop coupling (i.e. undesired transients of $e_{i-1}$ ) is caused by the control action $u_{i}$ to compensate $e_{i}$. In fact, as shown in (Cantoni et al., 2007; Li et al., 2005), in purely decentralized feedback control for a string of identical pools, where the local controllers are selected the same for each pool, there exists the following trade-off between local performance and the coupling between the pools: ${ }^{3}$

$$
T_{r_{i} \mapsto e_{i}}+T_{e_{i} \mapsto e_{i-1}} e^{-s \tau_{i}}=1,
$$

where $T_{r_{i} \mapsto e_{i}}=s \alpha_{i} T_{d_{i} \mapsto e_{i}}$. Indeed, similar performance trade-off exists for the control of a string of non-identical pools. In (Cantoni et al., 2007; Li and Cantoni, 2008), a distributed controller is synthesized by solving a structured $\mathcal{H}_{\infty}$ optimization problem to cope with the above identified trade-off.

\subsection{Distributed controller design}

Fig. 2 shows an irrigation channel with a special structured distributed control, i.e. the information flow is uni-directional: from controller $K_{i+1}$ to controller $K_{i}$. When water offtakes occur in a pool, such an interconnection structure confines the water-level error propagation and the amplification of control

\footnotetext{
${ }^{2}$ Where it is clear from the context, $y_{i}$ (instead of $y_{i}(s)$ ) will be used to denote the Laplace transform of $y_{i}(t)$. The same convention holds for $d_{i}, u_{i}$, etc.

${ }^{3}$ Let $T_{r_{i} \mapsto e_{i}}$ denote the transfer function from $r_{i}$ to $e_{i}$ and $T_{e_{i} \mapsto e_{i-1}}$ the transfer function for the water-level error propagation from pool $_{i}$ to pool $_{i-1}$.
} 
actions in the upstream pools. Hence, the requirement of water storage at the downstream end of the channel is avoided.

The localized portion of a channel of $N$ pools under the distributed distantdownstream control is shown in Fig. 3 . In the figure, $P_{i}$ is the nominal model

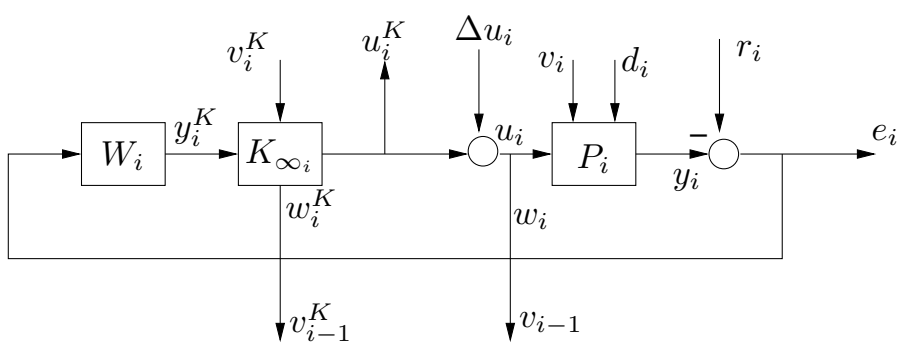

Figure 3: Localized portion of distributed controller design

(2) for $\operatorname{pool}_{i}$, and $K_{i}$ in Fig. 2 is split into a loop-shaping weight $W_{i}$ and a compensator $K_{\infty_{i}}$ (with $u_{i}^{K}$ and $y_{i}^{K}$, the input to and the output from the shaped plant, respectively). Note the constraint on the information flow structure of the distributed controller

$$
v_{i}^{K}=w_{i+1}^{K},
$$

where $v_{i}^{K}$ is the interconnection input of sub-controller $K_{i}$ and $w_{i+1}^{K}$ is the interconnection output of controller $K_{i+1}$. Designing the distributed controller consists of the following three steps, which is consistent with the well-known $\mathcal{H}_{\infty}$ loop-shaping approach (McFarlane and Glover, 1990):

1. Design $W_{i}$ to shape $P_{i}$ based on local performance.

Typically offtakes $d_{i}$ are step disturbances; based on the internal model principle (Goodwin et al., 2001), for a zero steady-state water-level error a simple selection of the shaping function could thus be

$$
W_{i}(s)=\frac{\kappa_{i}}{s} .
$$

For robust stability, $\kappa_{i}$ is selected such that the local crossover frequency $\omega_{c_{i}}$ is less than $1 / \tau_{i}$ (see Skogestad and Postlethwaite, 1996). Moreover, to ensure no excitement of dominant waves that are not captured by the first-order model (1), the loop gain of the shaped plant must be set to be low around the frequency of the local wave dynamics, see Section 4.1 for an example.

Denote $n_{i}:=\left(r_{i}, \Delta u_{i}, d_{i}\right)^{T}$ and $z_{i}:=\left(e_{i}, u_{i}^{K}\right)^{T}$, with $\Delta u_{i}$ modeling an additional disturbance on the flow at gate ${ }_{i}$. Each shaped sub-plant can then be represented as

$$
\begin{aligned}
& G_{s_{i}}:=\left(\begin{array}{c}
v_{i} \\
n_{i} \\
u_{i}^{K}
\end{array}\right) \mapsto\left(\begin{array}{c}
w_{i} \\
z_{i} \\
y_{i}^{K}
\end{array}\right) \\
& =\left[\begin{array}{ccc}
0 & \left(\begin{array}{ccc}
0 & 1 & 0
\end{array}\right) \\
\left(\begin{array}{ccc}
\frac{1}{s \alpha_{i}} \\
0
\end{array}\right. & \left(\begin{array}{ccc}
1 & \frac{e^{-s \tau_{i}}}{-s \alpha_{i}} & \frac{1}{s \alpha_{i}} \\
0 & 0 & \left(\begin{array}{c}
\frac{e^{-s \tau_{i}}}{-s \alpha_{i}}
\end{array}\right) \\
\frac{W_{i}}{s \alpha_{i}} & \left(W_{i} \frac{e^{-s \tau_{i} W_{i}}}{-s \alpha_{i}}\right. & \frac{W_{i}}{s \alpha_{i}}
\end{array}\right) & \frac{e^{-s \tau_{i}} W_{i}}{-s \alpha_{i}}
\end{array}\right]
\end{aligned}
$$


with $v_{i}=w_{i+1}$. Let $G_{s}:=\left(G_{s_{1}}, \ldots, G_{s_{N}}\right)$ denote the interconnection of the $G_{s_{i}}$ 's. Note that $G_{s_{N}}:=\left(\begin{array}{l}n_{N} \\ u_{N}^{K}\end{array}\right) \mapsto\left(\begin{array}{l}w_{i} \\ z_{N} \\ y_{N}^{K}\end{array}\right)$, i.e it is assumed that $v_{N}=0$. Such a boundary condition, i.e. no flow over the downstream gate of $\operatorname{pool}_{N}$, is possible with distant-downstream control (Cantoni et al., 2007). Indeed, this is important since water that flows out over the last gate is usually wasted.

2. Synthesize $K_{\infty_{i}}$ to cope with the trade-off between local performance and closed-loop coupling.

Let $K_{\infty}:=\left(K_{\infty_{1}}, \ldots, K_{\infty_{N}}\right)$ denote the interconnection of $K_{\infty_{i}}$ 's with $v_{i}^{K}=w_{i+1}^{K}$ and boundary condition $v_{N}^{K}=0$; and let $H\left(G_{s}, K_{\infty}\right)$ denote the closed-loop transfer function from $\left(n_{1}, \ldots, n_{N}\right)^{T}$ to $\left(z_{1}, \ldots, z_{N}\right)^{T}$. The synthesis problem is formulated as

$$
\begin{aligned}
& \min _{K_{\infty} \in \mathcal{K}_{\text {syn }}} \gamma \\
& \text { subject to } \\
&\left\|H\left(G_{s}, K_{\infty}\right)\right\|_{\infty}<\gamma
\end{aligned}
$$

where $\mathcal{K}_{\text {syn }}$ represents the set of stabilizing $K_{\infty}$ 's. Note that $\|\cdot\|_{\infty}$ denotes the $\mathcal{H}_{\infty}$ norm of a transfer function. Such a structured optimization problem can be solved by employing the technique in (Langbort et al., 2004), which investigates a special class of integral quadratic constraints to model the interconnection links of the plant, see (Li and Cantoni, 2008). The synthesized controller is denoted as

$$
K_{\infty_{i}}:=\left(\begin{array}{c}
v_{i}^{K} \\
y_{i}^{K}
\end{array}\right) \mapsto\left(\begin{array}{c}
w_{i}^{K} \\
u_{i}^{K}
\end{array}\right)=\left[\begin{array}{ll}
K_{\infty_{i}}^{11} & K_{\infty_{i}}^{12} \\
K_{\infty_{i}}^{21} & K_{\infty_{i}}^{22}
\end{array}\right]
$$

3. The final distributed controller is given by

$$
\begin{aligned}
K_{i} & :=\left(\begin{array}{c}
v_{i}^{K} \\
e_{i}
\end{array}\right) \mapsto\left(\begin{array}{c}
w_{i}^{K} \\
u_{i}
\end{array}\right) \\
& =K_{\infty_{i}}\left[\begin{array}{cc}
1 & 0 \\
0 & W_{i}
\end{array}\right]=\left[\begin{array}{cc}
K_{\infty_{i}}^{11} & K_{\infty_{i}}^{12} W_{i} \\
K_{\infty_{i}}^{21} & K_{\infty_{i}}^{22} \\
W_{i}
\end{array}\right],
\end{aligned}
$$

which combines the synthesized $K_{\infty_{i}}$ and the shaping function $W_{i}$.

Remark 1. A better global performance is anticipated by selecting the distributed controller configuration in Fig. 3, i.e. the position of $W_{i}$ and that of $K_{\infty_{i}}$ are swapped, compared to the localized portion of the distributed controller configuration suggested in (Cantoni et al., 2007; Li and Cantoni, 2008). Note that based on the distributed controller configuration in Fig. 3, in the synthesis of $K_{\infty_{i}}$ (i.e. the formulation of the optimization problem (3)), one of the output signal, $u_{i}^{K}$, is the flow at gate ${ }_{i}$; while with that of (Cantoni et al., 2007; $\mathrm{Li}$ and Cantoni, 2008), $u_{i}^{K}$ is a function of the flow at gate ${ }_{i}$ (with the physical interpretation unclear). 
Remark 2. Another weight structure, i.e. a PI compensator with a low pass filter, has been suggested in (Cantoni et al., 2007; Li and Cantoni, 2008). The performance of the final distributed controller is similar as the one using the simple weight in this paper (i.e. an integrator). Note that with an integrator as the weight, the final distributed controller is a 4th-order system: one state is from the weight, the other three from the synthesized part; while with a PI compensator with low-pass filter as the weight, the final distributed controller is a 6th-order system: two states from the weight, the other four from the synthesized part. So, to decrease the order of the final distributed controller, we suggest using an integrator as the shaping weight.

Remark 3. As pointed out in Section 1, this paper focuses on analyzing the decoupling function of the distributed controller introduced above. Note that in ( $\mathrm{Li}$ and De Schutter, 2010), the design of an extra feedforward compensator in addition to the existing distributed controller is explored, which improves the local control performance while maintaining the decoupling property of distributed control.

\subsection{Field test results}

The above introduced distributed control scheme has been tested in the field - specially, on five pools of Coleambally Channel Number 6, New South Wales, Australia. Fig. 4 shows the overshot gates working on the channel. The

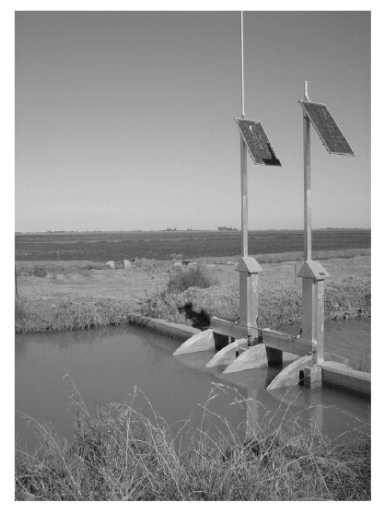

Figure 4: Overshot gates on Coleambally Channel Number 6 (Source: Rubicon Systems Australia Pty. Ltd)

identified model parameters are given in Table I (Ooi et al., 2001). ${ }^{4}$ See (Li and Cantoni, 2008) for the details of the design of the distributed controller. The test data are shown in Fig. 5, which provides both water-levels and gate flows over a period of 12 hours.

\footnotetext{
${ }^{4}$ In the system identification of the irrigation channel, the measurements of water-levels and gate positions are uniformly sampled with a period of 1 minute. To be consistent with the literature (see e.g., (Weyer, 2001)), we use 'min' as unit for time.
} 


\begin{tabular}{llllll}
\hline \hline pool & 1 & 2 & 3 & 4 & 5 \\
\hline$\tau_{i}(\min )$ & 4 & 2 & 4 & 4 & 6 \\
$\alpha_{i}\left(\mathrm{~m}^{2}\right)$ & 6492 & 2478 & 6084 & 5658 & 7650 \\
$\psi_{i}(\mathrm{rad} / \mathrm{min})$ & 0.48 & 1.05 & 0.48 & 0.48 & 0.42 \\
\hline
\end{tabular}

Table 1: Delay $\left(\tau_{i}\right)$, surface area $\left(\alpha_{i}\right)$ and dominant wave frequency $\left(\psi_{i}\right)$ extrapolated from validated system identification models (for the 5 pools in Coleambally Channel Number 6 )
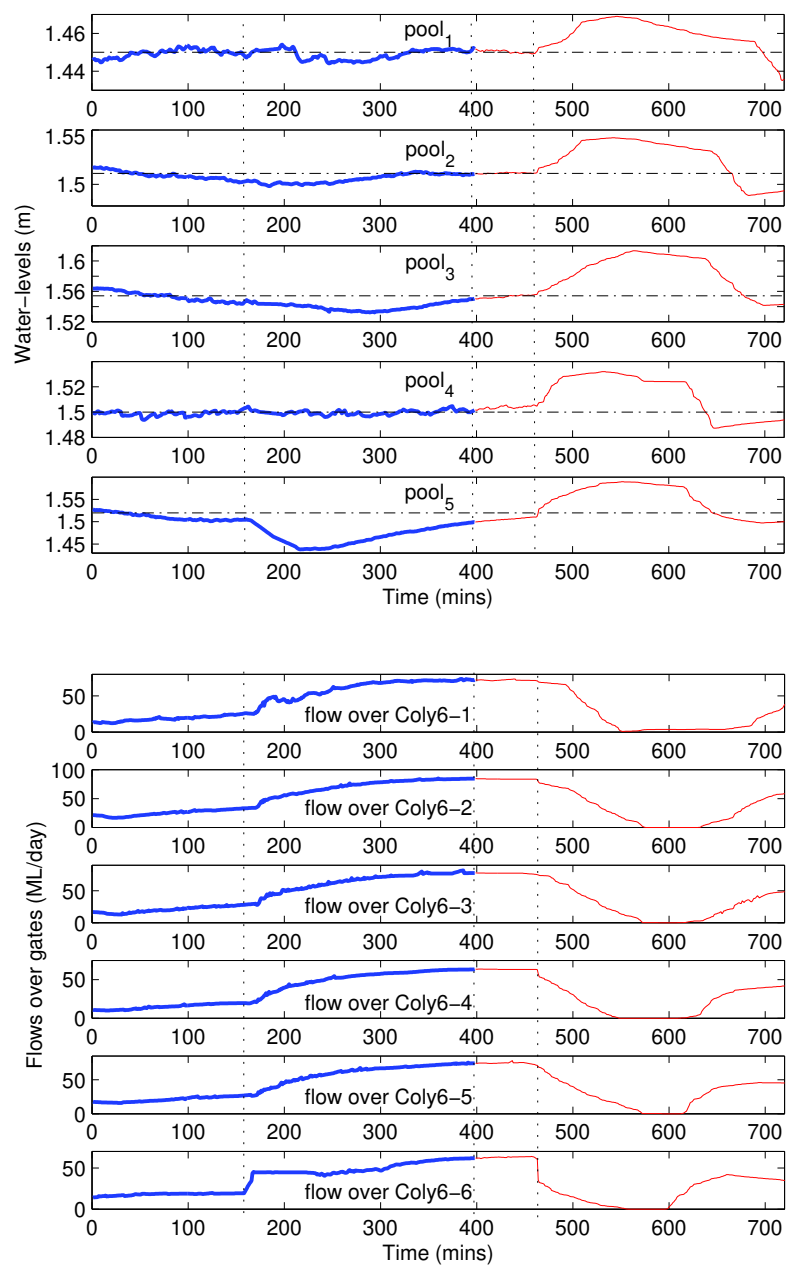

Figure 5: Field test data (Water levels in pool $_{1, \ldots .5}$ and flows over the gates: Coly6-1 through Coly6-6, on Coleambally Channel Number 6, New South Wales, Australia)

The water-level setpoints were set to $r_{1}=1.450 \mathrm{~m}, r_{2}=1.510 \mathrm{~m}, r_{3}=$ $1.554 \mathrm{~m}, r_{4}=1.500 \mathrm{~m}$ and $r_{5}=1.520 \mathrm{~m}$. Before $398 \mathrm{~min}$ (thick solid lines), the 
upstream gates of the five pools, Coly6-1 through Coly6-5, are controlled by the distributed controller. At $398 \mathrm{~min}$, control is transferred, with the assistance of a bumpless-transfer compensation, ${ }^{5}$ to a decentralized controller with additional decentralized feed-forward action (Weyer, 2008). To perturb the system, the flow over the downstream gate of pool $_{5}$, Coly6-6, is manually increased from 20 $\mathrm{Ml} /$ day to $45 \mathrm{Ml}$ /day at $159 \mathrm{~min}$ and kept increasing to $73 \mathrm{Ml} /$ day till $462 \mathrm{~min}^{6}$. This offtake load-disturbance is ceased at $462 \mathrm{~min}$, which perturbs the system again. The performance of the optimal distributed controller is clearly superior to decentralized feedback control with feedforward compensation in decoupling the interaction between pools. ${ }^{7}$ Indeed, with distributed control, the maximum water-level errors in the upstream four pools are around $0.01 \mathrm{~m}$ when the offtake disturbance works in pool $_{5}$; while with decentralized feedback with feedforward compensation, the water-level errors in the upstream pools go up to $0.07 \mathrm{~m}$. Moreover, note that when the offtake is removed the decentralized controller causes gate saturations (e.g. gate Coly6-4 reaches the maximum limits from 581 $\min$ to $599 \mathrm{~min})$.

In the next two sections, the impact of the internal time-delays on closedloop stability and performance is analyzed. Further, an investigation is made into how the decoupling terms in the distributed controller compensate such an impact.

\section{The impact of the internal time-delays on closed-loop performance}

For distant-downstream control, the internal time-delay $\tau_{i}$ limits the local performance. This is understandable, since no matter what controller is being used, the effect of an input change on the output will be delayed by the time $\tau_{i}$. Furthermore, as discussed in Section 2.3, for robust stability of the closed-loop system, the local bandwidth limit of $1 / \tau_{i}$ is considered in the selection of the weight gain, $\kappa_{i}$. In this section, the influences of $\tau_{i}$ on the closed-loop coupling are discussed. It is shown that such time-delays do not only make it difficult to manage the water-level error propagation, but also cause the amplification of control actions in the upstream direction.

\footnotetext{
${ }^{5}$ A strategy of designing distributed anti-windup compensator is discussed in (Li and Cantoni, 2007), which also provides bumpless transients in the face of control switches.

${ }^{6}$ To follow the typical parameter-units in the Australian irrigation districts where the field trials have been conducted, 'Ml/day' is used in the paper as the unit for flows. Note that $1 \mathrm{Ml} /$ day $=0.0116 \mathrm{~m}^{3} / \mathrm{sec}$.

${ }^{7}$ In fact, the decentralized feedback control with feedforward compensation in (Weyer, 2008 ) is a special realization of the distributed controller, i.e. $K_{i}=\left[\begin{array}{l}F_{i} C_{i} \\ F_{i} C_{i}\end{array}\right]$, where $F_{i}$ is the feedforward part to decouple the interaction between pools and $C_{i}$ the feedback part to stabilize the system and to deal with model errors and unknown perturbations.
} 


\subsection{Stability and robustness analysis}

From (2), for a channel of $N$ pools

$$
\begin{aligned}
\left(\begin{array}{c}
y_{1} \\
y_{2} \\
\vdots \\
y_{N-1} \\
y_{N}
\end{array}\right) & =\left[\begin{array}{ccccc}
G_{1} & \tilde{G}_{1} & 0 & \cdots & 0 \\
0 & G_{2} & \tilde{G}_{2} & \ddots & \vdots \\
\vdots & \ddots & \ddots & \ddots & 0 \\
0 & \cdots & 0 & G_{N-1} & \tilde{G}_{N-1} \\
0 & \cdots & \cdots & 0 & G_{N}
\end{array}\right]\left(\begin{array}{c}
u_{1} \\
u_{2} \\
\vdots \\
u_{N-1} \\
u_{N}
\end{array}\right) \\
+ & {\left[\begin{array}{cccc}
\tilde{G}_{1} & 0 & \cdots & 0 \\
0 & \tilde{G}_{2} & \ddots & \vdots \\
\vdots & \ddots & \ddots & 0 \\
0 & \cdots & 0 & \tilde{G}_{N}
\end{array}\right]\left(\begin{array}{c}
d_{1} \\
d_{2} \\
\vdots \\
d_{N}
\end{array}\right) }
\end{aligned}
$$

where $G_{i}=\frac{1}{s \alpha_{i}} e^{-s \tau_{i}}$ and $\tilde{G}_{i}=-\frac{1}{s \alpha_{i}}$. As mentioned in Section 2.3, it is reasonable to assume $v_{N}=0$ as boundary condition for synthesis of the distributed controller under distant-downstream control. The distributed controller is represented by

$$
\begin{array}{rll}
K_{1}: & u_{1}=\left[\begin{array}{ll}
K_{1}^{21} & K_{1}^{22}
\end{array}\right]\left(\begin{array}{c}
w_{2}^{K} \\
e_{1}
\end{array}\right) \\
K_{i} & : \quad\left(\begin{array}{c}
w_{i}^{K} \\
u_{i}
\end{array}\right)=\left[\begin{array}{ll}
K_{i}^{11} & K_{i}^{12} \\
K_{i}^{21} & K_{i}^{22}
\end{array}\right]\left(\begin{array}{c}
w_{i+1}^{K} \\
e_{i}
\end{array}\right) \text { for } i=2, \ldots, N-1 \\
K_{N} & : \quad\left(\begin{array}{c}
w_{N}^{K} \\
u_{N}
\end{array}\right)=\left[\begin{array}{l}
K_{N}^{12} \\
K_{N}^{22}
\end{array}\right] e_{N} .
\end{array}
$$

This gives the general form of the distributed controller $K$ :

$$
\left(\begin{array}{c}
u_{1} \\
u_{2} \\
\vdots \\
u_{N}
\end{array}\right)=\left[\begin{array}{cccc}
K_{11} & K_{12} & \cdots & K_{1 N} \\
0 & K_{22} & \cdots & K_{2 N} \\
\vdots & \ddots & \ddots & \vdots \\
0 & \cdots & 0 & K_{N N}
\end{array}\right]\left(\begin{array}{c}
e_{1} \\
e_{2} \\
\vdots \\
e_{N}
\end{array}\right)
$$

where for $i=1, \ldots, N$,

$$
K_{i i}=K_{i}^{22}
$$

which takes care of local performance, and the additional decoupling terms

$$
\begin{aligned}
K_{i, i+1} & =K_{i}^{21} K_{i+1}^{12} \\
K_{i j} & =K_{i}^{21}\left(\prod_{k=i+1}^{j-1} K_{k}^{11}\right) K_{j}^{12} \text { for } j>i+1 .
\end{aligned}
$$

From (6) and (7), the closed-loop relationship between water-level errors and offtake disturbances is:

$$
\left(\begin{array}{c}
e_{1} \\
e_{2} \\
\vdots \\
e_{N}
\end{array}\right)=\left[\begin{array}{cccc}
M_{11} & M_{12} & \cdots & M_{1 N} \\
0 & M_{22} & \cdots & M_{2 N} \\
\vdots & \ddots & \ddots & \vdots \\
0 & \cdots & 0 & M_{N N}
\end{array}\right]\left(\begin{array}{c}
d_{1} \\
d_{2} \\
\vdots \\
d_{N}
\end{array}\right)
$$


where for $i=1, \ldots, N, M_{i i}=-\tilde{G}_{i}\left(1+G_{i} K_{i i}\right)^{-1}$ and for $j \geq i+1$

$$
M_{i j}=M_{i i} \sum_{k=i+1}^{j}\left(K_{i+1, k}-K_{i k} e^{-s \tau_{i}}\right) M_{k j}
$$

It is noted that the closed-loop transfer matrix is upper-triangular, hence the multivariable system inherits the local stabilities, i.e. the multivariable system is stable if and only if all monovariable systems are stable. Since all the lower offdiagonal entries are null, even for model mismatch, robustness is also inherited from the local closed-loop systems.

It is direct from (10) and (11) that a perfect decoupling is achieved if for all $j>i$,

$$
K_{i+1, j}-K_{i j} e^{-s \tau_{i}}=0
$$

This requires $K_{i j}=K_{i+1, j} e^{s \tau_{i}}$, which is non-causal and hence impractical. In Section 3.2, analyzes are made on the impacts of the internal time-delays on the two typical coupling properties of a (distant-downstream) controlled irrigation channel: water-level error propagation and amplification of control actions, respectively.

\subsection{Global closed-loop performance}

Here, the worst-case properties of the coupling transfer functions, i.e. $\left\|T_{e_{i+1} \mapsto e_{i}}\right\|_{\infty}$ and $\left\|T_{u_{i+1} \mapsto u_{i}}\right\|_{\infty}$, are explored. As discussed in (Welz et al., 2005), since in the case of irrigation channels the perturbations are unknown, the maximum singular value of the closed-loop coupling transfer functions are a good estimate of the coupling properties of the controlled channel.

\subsubsection{Water-level error propagation}

From (10), the water-level errors in $\operatorname{pool}_{i}$ and $\operatorname{pool}_{i+1}$ in response to the load-disturbances $d_{j}$ (i.e. the offtakes occurring in $\operatorname{pool}_{j}$ ), for $j \geq i+1$, have the following relationship:

$$
\begin{aligned}
& T_{e_{i+1} \mapsto e_{i}}:=M_{i, j} M_{i+1, j}^{-1} \\
& =M_{i i}\left(K_{i+1, i+1}-e^{-s \tau_{i}} K_{i, i+1}\right)+M_{i i} \sum_{k=i+2}^{j}\left(K_{i+1, k}-K_{i k} e^{-s \tau_{i}}\right) M_{k j} \\
& \left(M_{i+1, i+1} \sum_{k=i+2}^{j}\left(K_{i+2, k}-K_{i+1, k} e^{-s \tau_{i+1}}\right) M_{k j}\right)^{-1}
\end{aligned}
$$

A small $\left\|T_{e_{i+1} \mapsto e_{i}}\right\|_{\infty}($ e.g. $\ll 1)$ represents a good management of the waterlevel error propagation.

Remark 4. For the case of a string of identical pools with purely decentralized feedback control (i.e. $\left.K=\operatorname{diag}\left(K_{i i}\right)\right), T_{e_{i+1} \mapsto e_{i}}=M_{i i} K_{i+1, i+1}$. If the selected 
$K_{i i}$ 's are identical for all $i=1, \ldots, N$, then $\left\|T_{e_{i+1} \mapsto e_{i}}\right\|_{\infty}>1$ (see the proof in (Cantoni et al., 2007; Li and De Schutter, 2010)). Such a strategy, i.e. designing $K_{i i}$ only based on local control performance, thus creates very strong coupling between loops (since $\left\|T_{e_{i+1} \mapsto e_{i}}\right\|_{\infty}$ occurs at the same frequency for all $i$ ). Instead, to decouple the interaction between pools, one can design $K_{i i}$ 's such that the downstream closed-loop is slower than the upstream ones ( $\mathrm{Li}$ and De Schutter, 2010). However, it is nontrivial to cope with the trade-off between local performance and closed-loop decoupling by simply tuning the feedback controller.

For simplicity, one considers two consecutive pools, $\operatorname{pool}_{i}$ and $\operatorname{pool}_{i+1}$, with distributed control. For an offtake disturbance $d_{i+1}$,

$$
\begin{aligned}
T_{e_{i+1} \mapsto e_{i}} & =M_{i, i+1} M_{i+1, i+1}^{-1} \\
& =M_{i i}\left(K_{i+1, i+1}-K_{i, i+1} e^{-s \tau_{i}}\right)
\end{aligned}
$$

Taking (5) and (8-9) into the right hand side of (13), it is direct to have that if

$$
K_{\infty_{i}}^{21} W_{i} K_{\infty_{i+1}}^{12} \approx K_{\infty_{i+1}}^{22} W_{i+1} e^{-s \tau_{i}}
$$

over the low and mid-frequency range of interest, then a good management of the water-level error propagation is achieved. If the two pools are identical, considering local performance, one can select $W_{i}=W_{i+1}$. The condition (14) is then simplified to that $\left(K_{\infty_{i}}^{21} K_{\infty_{i+1}}^{12}\right)^{-1} K_{\infty_{i+1}}^{22}$ is a lead-lag filter approximating $e^{\tau_{i} s}$ over the low and mid-frequency range.

\subsubsection{Control action amplification}

From (7) and (10), the coupling of control actions $u_{i+1}$ and $u_{i}$ (responding to $d_{j}$ for $\left.i \leq j-1\right)$ is

$$
T_{u_{i+1} \mapsto u_{i}}:=\sum_{k=i}^{j} K_{i k} M_{k j}\left(\sum_{k=i+1}^{j} K_{i+1, k} M_{k j}\right)^{-1} .
$$

The following discussions show that $\left\|T_{u_{i+1} \mapsto u_{i}}\right\|_{\infty}>1$ if the internal time-delay $\tau_{i}>0$.

Remark 5. For an irrigation channel with purely decentralized feedback control, i.e. $K$ in (7) being diagonal,

$$
T_{u_{i+1} \mapsto u_{i}}=M_{i i} K_{i i}=-\tilde{G}_{i} K_{i i}\left(1-\tilde{G}_{i} K_{i i} e^{-\tau_{i} s}\right)^{-1} .
$$

Note that $\tilde{G}_{i} K_{i i}$ involves two integrators. ${ }^{8}$ Applying Lemma 9.3 of (Goodwin et al., 2001), it is straightforward to prove $\left\|T_{u_{i+1} \mapsto u_{i}}\right\|_{\infty}>1$.

\footnotetext{
${ }^{8}$ From (8) and (5), $K_{i i}=K_{\infty_{i}}^{22} W_{i}$, where $W_{i}$ is selected to involve an integrator for zero steady-state water-level error, as discussed in Section 2.3.
} 
Generally, under distant-downstream control (i.e. without the constraints that $K$ in (7) be diagonal), to compensate the influence of the internal timedelay, the amplification of control action in the upstream direction is unavoidable. This is shown in Fig. 6. Initially, the system is at steady-state. At time

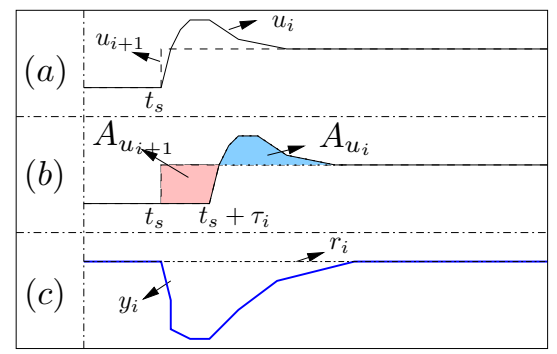

Figure 6: Control actions for zero steady-state water-level error

$t_{s}$, the flow out of pool $_{i}$ increases, see the change of $u_{i+1}$ (the dashed line in Fig. 6(a)). To compensate for the influence of $u_{i+1}$ on $y_{i}$, the flow into the pool, $u_{i}$, also increases (the solid line in Fig. 6(a)). However, the influence of $u_{i}$ on the downstream water-level $y_{i}$ will be $\tau_{i}(\min )$ later than that of $u_{i+1}$ on $y_{i}$ (see Fig. 6(b)). For zero steady-state error of $y_{i}$ from $r_{i}$ (see Fig. 6(c)), from (2), $u_{i}$ should be greater than $u_{i+1}$ for some time such that the area of $A_{u_{i}}$ is equivalent to the area of $A_{u_{i+1}}$. Hence, $\left\|T_{u_{i+1} \mapsto u_{i}}\right\|_{\infty}>1$.

Indeed, under the distributed control scheme, in the face of $d_{i+1}$, the following relationship between $u_{i}$ and $u_{i+1}$ exists:

$$
\begin{aligned}
T_{u_{i+1} \mapsto u_{i}} & =\left(K_{i i} M_{i, i+1}+K_{i, i+1} M_{i+1, i+1}\right) M_{i+1, i+1}^{-1} K_{i+1, i+1}^{-1} \\
& =K_{i i} M_{i i}\left(1-K_{i, i+1} K_{i+1, i+1}^{-1} e^{-s \tau_{i}}\right) \\
& +K_{i, i+1} K_{i+1, i+1}^{-1} .
\end{aligned}
$$

If condition (14) is satisfied around the low and mid-frequency range, then directly from (15) one has $\left\|T_{u_{i+1} \mapsto u_{i}}\right\|_{\infty} \gtrsim 1$.

\section{Decoupling property of the distributed controller - a case study}

In this section, a simulation is done on a string of three pools to investigate the properties of the decoupling terms in the distributed controller in compensating the impact of time-delays on the global closed-loop performance.

The three pools are taken from the Eastern Goulburn Channel Number 12, Victoria, Australia. Table II gives the identified model parameters (Ooi et al., 2001). The geometric properties of the pools are also provided.

Remark 6. For further analysis, a case that is different from the one given in Section 2.4 is selected. From the perspective of offtake rejection, the field test case (i.e. a string of pools in Coleambally Channel No. 6) of Section 2.4 is simple 


\begin{tabular}{llll}
\hline \hline pool & 1 & 2 & 3 \\
\hline$\tau_{i}(\min )$ & 6 & 25 & 15 \\
$\alpha_{i}\left(\mathrm{~m}^{2}\right)$ & 10344 & 39352 & 26317 \\
$\psi_{i}(\mathrm{rad} / \mathrm{min})$ & 0.349 & 0.084 & 0.140 \\
Length $(\mathrm{m})$ & 1350 & 5000 & 3420 \\
Bottom width $(\mathrm{m})$ & 5.65 & 5.71 & 5.66 \\
Side slope & 2.5 & 2.5 & 2.5 \\
Bottom slope $\times 10^{-4}$ & 1.5 & 1.5 & 1.5 \\
Upstream gate width $(\mathrm{m})$ & 4.6938 & 4.6740 & 4.6928 \\
Downstream gate width $(\mathrm{m})$ & 4.6740 & 4.6928 & 6.0720 \\
\hline
\end{tabular}

Table 2: Delay $\left(\tau_{i}\right)$, surface area $\left(\alpha_{i}\right)$ and dominant wave frequency $\left(\psi_{i}\right)$ extrapolated from validated system identification models (for the 3 pools on Eastern Goulburn Channel Number 12)

for control design given that the pools are short (i.e. from 0.4 to 1.3 kilometer) and the dynamics of the pools slow down from upstream to downstream. On the other hand, the string of pools in Eastern Goulburn channel No. 12 selected in this section (with pool length from 1.7 to 6.7 kilometer) represents one of the most challenging cases to control in terms of global performance, since the middle pool $\left(\mathrm{pool}_{2}\right)$ has a much larger internal time-delay than the downstream pool $\left(\mathrm{pool}_{3}\right)$. Under decentralized feedback control, when an offtake occurs in pool $_{3}$, because of the big $\tau_{2}$ a large transient of $u_{2}$ (i.e. the flow deviation from steady-state over the upstream gates of $\mathrm{pool}_{2}$ ) to compensate $e_{2}$ is observed, although a small transient of $u_{3}$ is achievable with $\tau_{3}$ being small. As discussed in Section 3.2.2, such an undesired transient will be amplified in the upstream direction and hence for a string of many pools, some saturation limits of the control actions in the upstream pools will be exceeded.

\subsection{Simulation results}

To shape the plant, choose $^{9} W_{1}=\frac{87.20}{s}, W_{2}=\frac{20.88}{s}, W_{3}=\frac{32.62}{s}$. A $\gamma=3$ is achieved by solving the structured optimization problem (3). The final controller is shown in Fig. 7. All the terms involve an integrator, which comes from the shaping weights. Note that $K_{12}$ has similar phase properties as $K_{22}$, i.e. they both involve phase-lead-lag-lag-lead compensation around the same mid-frequency range; while $K_{13}, K_{23}$ have similar phase properties as $K_{33}$.

Fig. 8 shows the open loop-gain for pool ${ }_{1,2,3}$. A high gain at low frequencies is obtained, with the bandwidths being $0.0408 \mathrm{rad} / \mathrm{min}, 0.0085 \mathrm{rad} / \mathrm{min}$, and $0.0132 \mathrm{rad} / \mathrm{min}$ respectively. Around the dominant wave frequencies (see $\psi_{i}$ in Table II), the loop-gains are about $-20 \mathrm{~dB},-20 \mathrm{~dB}$ and $-25 \mathrm{~dB}$ respectively. This ensures no excitement of dominant waves in all the three pools.

\footnotetext{
${ }^{9}$ As formerly discussed, the weight gains are chosen to set the loop-gain bandwidth just below $1 / \tau_{i}$.
} 


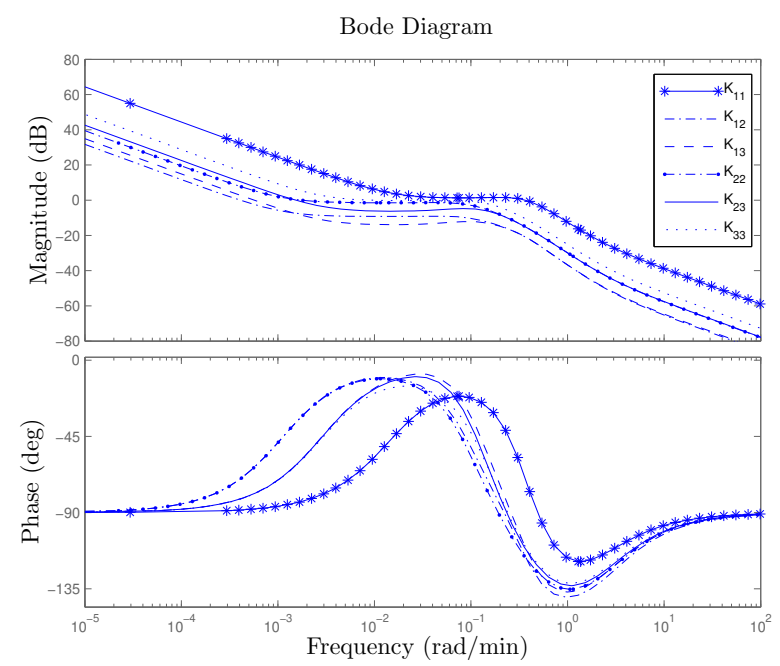

Figure 7: The distributed controller

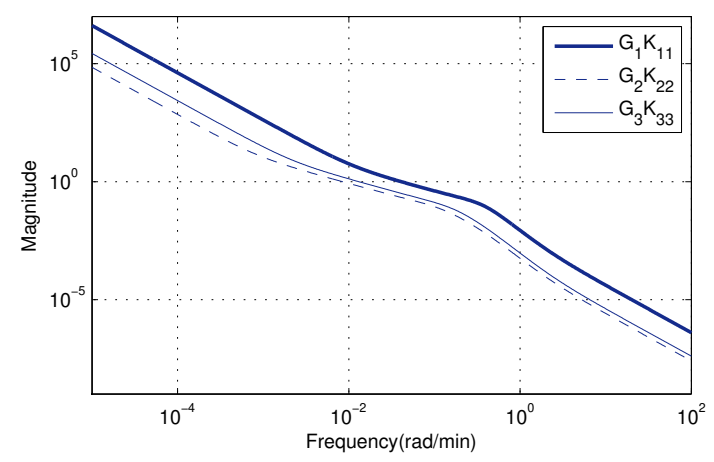

Figure 8: Local loop-gain with the distributed controller

The behavior of the control system was simulated using the Saint Venant equations model for the string of pools, which is a more accurate quantitative model than the integrator with time-delay model used for controller design. In the simulation, the system is initially at steady-state, i.e. the water-levels in the three pools are at their setpoints $r_{i}=1.8 \mathrm{~m}$ for $i=1,2,3$, and the flows over gates $^{10} u_{i}=100 \mathrm{Ml} /$ day for $i=1, \ldots, 4$. To perturb the system, an offtake of $113 \mathrm{Ml} /$ day starts in $\mathrm{pool}_{3}$ at $30 \mathrm{~min}$ (i.e. $d_{3}$ ). Such an offtake works on the system till the end of the simulation scenario (i.e. 2000 min). Fig. 9 shows the

${ }^{10}$ Note $u_{4}$ is the flow over the downstream gate of pool $_{3}$. 
simulated water-level errors and flows over upstream gates of the three pools, when

- operating under a decentralized controller (Cantoni et al., 2007), where the isolated local compensator for pool $_{i}$ achieves similar open loop gain as that shown in Fig. 8, and when

- operating under the distributed controller designed above (with frequencydomain properties shown in Fig. 7).

Compared with decentralized control, the distributed controller clearly achieves a significantly improved global performance, while yielding a very similar local load-disturbance rejection performance (see $e_{3}$ and $u_{3}$ in Fig. 9 for both control structures).
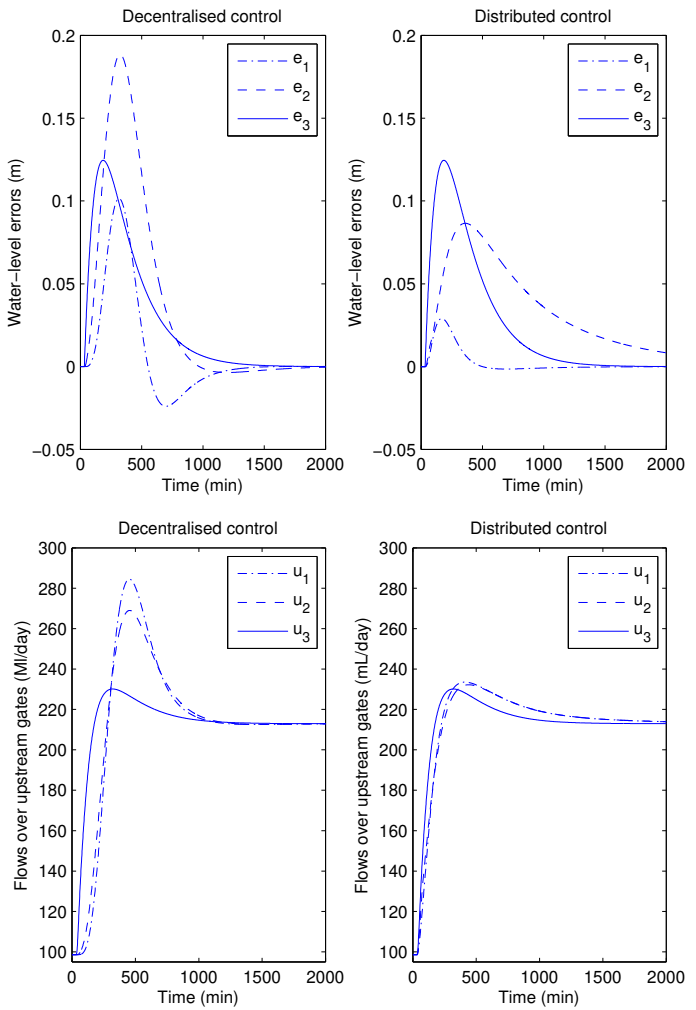

Figure 9: Simulated water-level errors and flows over upstream gates

As discussed in Section 2.3, the synthesis of $K_{\infty}$ (i.e. the formulation of the structured optimization problem (3)) copes with the trade-off between the local performance and the decoupling of the closed-loop system. To see how the distributed controller compensates for the influence of internal time-delays, 
the properties of the decoupling terms in the distributed controller are analyzed next.

\subsection{The influence of $K_{i j}(j>i)$ on closed-loop decoupling}

From (9), $K_{12}$ and $K_{23}$ have a similar structure, while $K_{13}$ involves $K_{2}^{11}$ for decoupling. The following analysis is made by checking the impact ${ }^{11}$ of $K_{23}$ and $K_{13}$ on the decoupling of the closed-loop system.

\subsubsection{Impact of $K_{23}$}

The gains of $T_{d_{3} \mapsto e_{2}}$ and $T_{d_{3} \mapsto u_{2}}$, with and without $K_{23}$, are given in Fig. 10 . With $K_{23}$, a lower gain in the mid-frequency range is achieved.
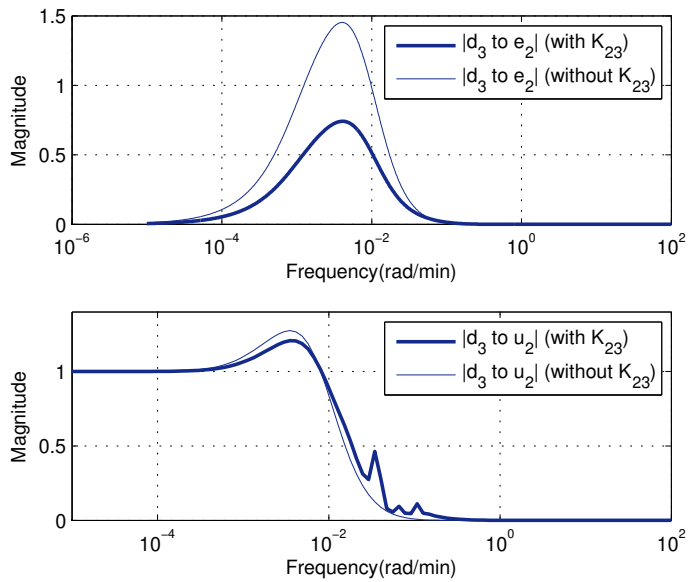

Figure 10: $\left|T_{d_{3} \mapsto e_{2}}\right|$ (top) and $\left|T_{d_{3} \mapsto u_{2}}\right|$ (bottom), with and without $K_{23}$

Fig. 11 shows that $K_{23}$ helps in decreasing $\left|T_{e_{3} \mapsto e_{2}}\right|$ and $\left|T_{u_{3} \mapsto u_{2}}\right|$ in the low and middle-frequency range, where $d_{3}$ is significant. One can thus expect a good management of the water-level error propagation and attenuation of the amplification of control action with $K_{23}$.

The time response of the closed-loop system is shown in Fig. 12. When $d_{3}$ starts at $30 \mathrm{~min}$, with $K_{23}$ (see the thick solid line), $\max _{t}\left|e_{2}(t)\right|$ decreases about $0.11 \mathrm{~m}$ compared with the case without $K_{23}$ (see the thin solid line). This is important since, as discussed in Section 1, in gravity-fed irrigation networks, water-levels represent the capacity to serve water-demands at the offtake points. Fig. 12(b) shows the upstream control actions in pool $_{2,3}$ to compensate the influence of $d_{3}$ on $e_{2}$ and $e_{3} \cdot{ }^{12}$ With $K_{23}, u_{2}$ responds to the change of $u_{3}$

\footnotetext{
${ }^{11} \mathrm{~A}$ similar impact of $K_{12}$ as that of $K_{23}$ on the closed-loop decoupling can be expected and hence the corresponding analysis is omitted here.

${ }^{12}$ For clarity, one zoomed in on the first 1000 mins to show the changes of the control actions when $d_{3}$ starts. Note this was also done in Fig. 14(b).
} 

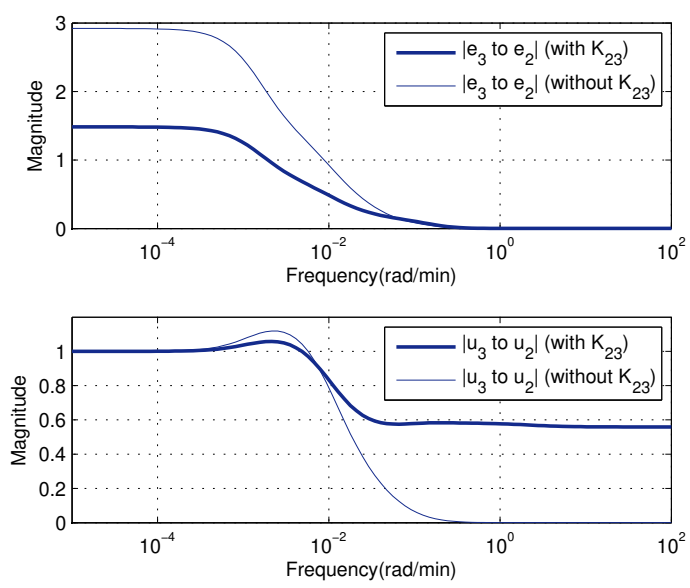

Figure 11: Closed-loop coupling: $\left|T_{e_{3} \mapsto e_{2}}\right|$ and $\left|T_{u_{3} \mapsto u_{2}}\right|$
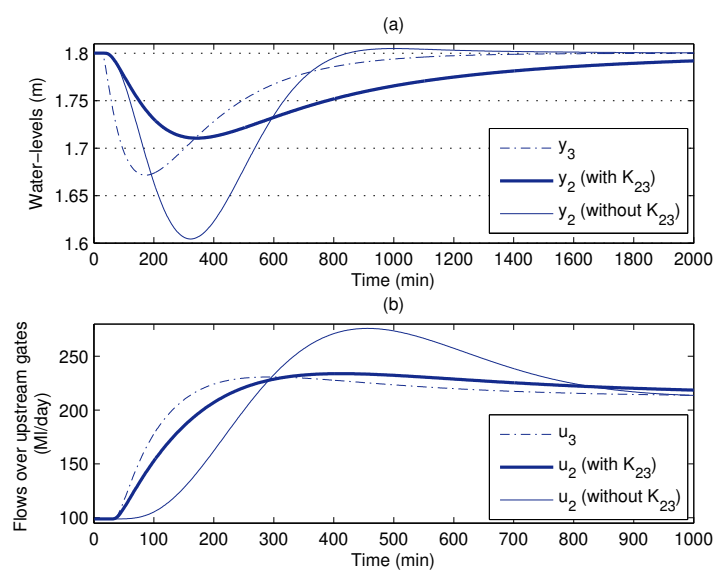

Figure 12: Time response of the closed-loop system: with and without $K_{23}$

faster than without $K_{23}$ operating on the closed-loop. Note $\max _{t}\left|u_{2}(t)\right|$ is much smaller with $K_{23}$ than without $K_{23}$, i.e. a better attenuation of the amplification of control action is obtained.

\subsubsection{Impact of $K_{13}$}

Fig. 13 shows $\left|T_{d_{3} \mapsto e_{1}}\right|$ and $\left|T_{d_{3} \mapsto u_{1}}\right|$, with and without $K_{13} \cdot{ }^{13}$ With $K_{13}$, a lower gain in the low and mid-frequency range is achieved, hence a better

\footnotetext{
${ }^{13}$ For the case of $K_{13}=0$, it is assumed that $K_{2}^{11}=0$, while $K_{12}$ and $K_{23}$ still operate on the closed-loop.
} 
decoupling of the closed-loop system can be expected. This is confirmed by the time responses shown in Fig. 14. When $d_{3}$ starts at $30 \mathrm{~min}$, the water-level error
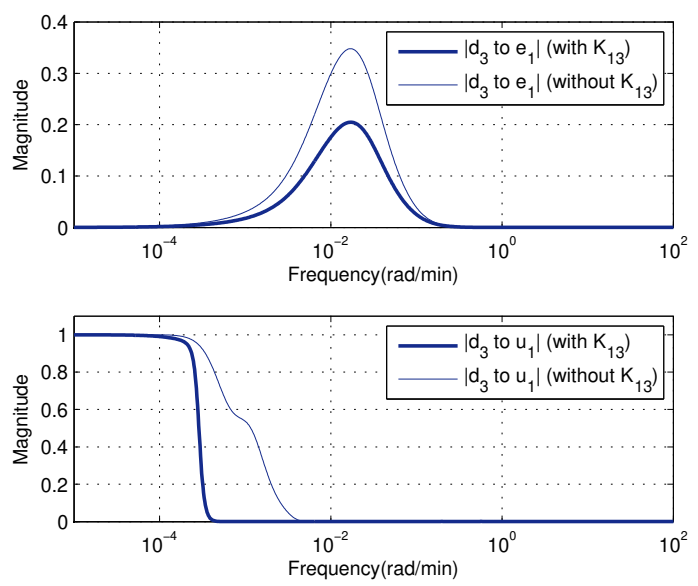

Figure 13: $\left|T_{d_{3} \mapsto e_{1}}\right|$ (top) and $\left|T_{d_{3} \mapsto u_{1}}\right|$ (bottom), with and without $K_{13}$

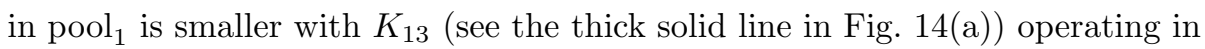
the system than without $K_{13}$ (the thin solid line). Fig. 14(b) shows the change
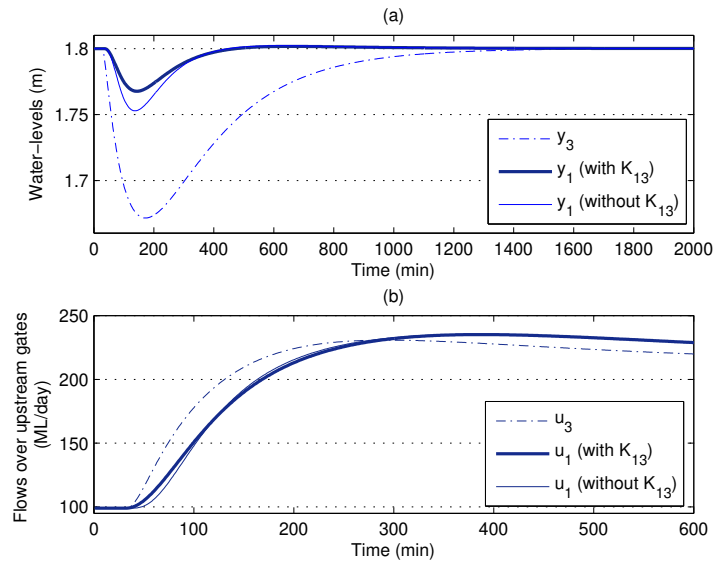

Figure 14: Time response of the closed-loop system: with and without $K_{13}$

of control actions in pool $_{1,3}$ in response to $d_{3}$. It is seen that with $K_{13}, u_{1}$ reacts (slightly) faster to the change in $u_{3}$ than the case without $K_{13}$.

\subsubsection{Further remarks}

The closed-loop coupling term $M_{i j}$ (see (11)) is composed of

$$
M_{i j}^{k}:=M_{i i}\left(K_{i+1, k}-K_{i k} e^{-s \tau_{i}}\right) M_{k j}
$$


for $k=i+1, \ldots, j$. Fig. 15 shows the impact of $K_{i k}$ on $M_{i j}^{k}$ in the above three-pool example. It is observed that

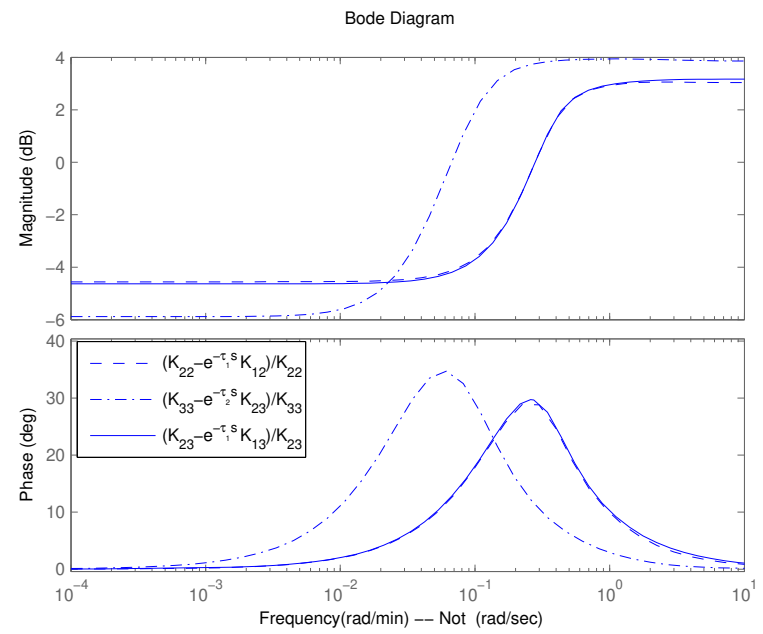

Figure 15: The decoupling function of $K_{i k}$ for $k=i+1, \ldots, j$

1. $K_{i k}$ decreases the gain of $M_{i j}^{k}$ at low frequencies where typical offtake disturbances are significant;

2. $K_{i k}$ operates on $M_{i j}^{k}$ by imposing on $M_{i i} K_{i+1, k} M_{k j}$ an additional phase lead-lag compensation around the frequency of $1 / \tau_{i}$.

Note that similar observations are made in other case studies for channels with number of pools $>3$. Indeed, the first observation explains why with $K_{i j}(j>i)$ operating on the closed-loop, a better management of water-level error propagation is achieved (see e.g. (13)). Although it is difficult to directly draw conclusions of global performance from the second observation, the following related observations can be made from the time-responses of control actions (see Fig. 12(b) and 14(b)):

- with the $K_{i j}$ 's (for $j>i$ ) the control actions in response to the offtake disturbance are faster than that without the $K_{i j}$ 's. Indeed, this is a result of the distributed control structure, i.e. with the decoupling terms, the control actions at downstream gates are fed forward and hence a faster response is expected.

- with the additional phase lead-lag compensation involved in the decoupling component $1-K_{i, i+1} K_{i+1, i+1}^{-1} e^{-s \tau_{i}}$, the peak components of the fast (or aggressive) control actions (induced by the distributed control structure), which might contribute to overshoot of the control actions, are attenuated. 


\section{Conclusions}

An irrigation channel is a system presenting strong interactions between pools. This paper has considered distant-downstream control of irrigation channels. It was shown that the internal time-delay for transportation of water from upstream to downstream of each pool does not only limit the local performance, but also impacts the coupling between pools, i.e. the water-level error propagation and the amplification of control actions in the upstream direction. More specifically, we have discussed a distributed control scheme that inherits the interaction structure of the plant. The controller is designed using a structured $\mathcal{H}_{\infty}$ loop-shaping approach. The optimization problem involved manages the trade-off between local and global performance. Analysis shows that the distributed controller compensates the time-delay influence by decreasing the low-frequency gain of the closed-loop coupling term and imposing extra phase lead-lag compensation in the mid-frequency range on the closed-loop coupling term.

It is of interest in future research to investigate distributed control with an even simpler interconnection structure, e.g. when the 11-block of each subcontroller (i.e. $K_{i}^{11}$ of sub-controller $i$ ) is set to 0 . Based on the analysis of the decoupling properties of the distributed controller (in compensating the internal time-delay) made in this paper, one might build up an elegantly structured controller that manages the trade-off between local and global performance for water networks.

\section{References}

Agriculture and Water (February 2010). Water Encyclopedia, Science and Issues, http://www.waterencyclopedia.com/A-Bi/Agriculture-and-water.html.

Cantoni, M., Weyer, E., Li, Y., Ooi, S. K., Mareels, I., Ryan, M. (2007). Control of large-scale irrigation networks. Bailliuel, J. and Antsaklis, P. (eds.), Special Issue on the Technology of Networked Control Systems, Proceedings of the IEEE, 95 (1), 75-91.

Clemmens, A. J., Schuurmans, J. (2004). Simple optimal downstream feedback canal controllers: theory. Journal of Irrigation and Drainage Engineering, 130 (1), 26-34.

Deltour, J. L. (1992). Application de l'automatique numérique à la régulation des canaux; Proposition d'une méthodologie d'étude, PhD Thesis, Institut National Polytechnique de Grenoble, in French.

Goodwin, G. C., Graebe, S., Salgado, M. (2001). Control System Design. Prentice Hall, Englewood Cliffs, NJ.

Langbort, C., Chandra, R., D’Andrea, R. (September 2004). Distributed control design for systems interconnected over an arbitrary graph. IEEE Transactions on Automatic Control, 49 (9), 1502-1519. 
Li, Y., Cantoni, M. (December 2007). On distributed anti-windup compensation for distributed linear control systems. In: Proceedings of the 46th IEEE CDC, New Orleans, LA, USA, pp. 1106-1111.

Li, Y., Cantoni, M. (July 2008). Distributed controller design for open water channels. In: Proceedings of the 17th IFAC World Congress, Seoul, South Korea, pp. 10033-10038.

Li, Y., Cantoni, M., Weyer, E. (December 2005). On water-level error propagation in controlled irrigation channels. In: Proceedings of the 44 th IEEE CDC-ECC'05, Seville, Spain, pp. 1502-1519.

Li, Y., De Schutter, B. (December 2010). Control of a string of identical pools using non-identical feedback controllers. In: Proceedings of the 49th IEEE CDC, Atlanta, Georgia, USA, pp. 120-125.

Li, Y., De Schutter, B. (2010). Offtake feedforward compensator design for irrigation channels with distributed control. Submitted to IEEE Transactions on Control Systems Technology.

Litrico, X., Fromion, V. (2003). Advanced control politics and optimal performance for an irrigation canal. In: Proceedings of the 2003 ECC, Cambridge, UK.

Litrico, X., Fromion, V. (December 2005). Design of structured multivariable controllers for irrigation canals. In: Proceedings of 44th IEEE CDC-ECC'05, Seville, Spain, pp. 1881-1886.

Malaterre, P. O. (1994). Modelisation, Analysis and LQR Optimal Control of an Irrigation Canal. Ph.D. Thesis, LAAS-CNRS-ENGREF-Cemagref, Etude EEE n14, ISBN 2-85362-368-8.

Malaterre, P. O., Baume, J. P. (1999). Optimum choice of control action variables and linked algorithms; Comparison of different alternatives. ASCEICID Workshop on Modernization of Irrigation Water Delivery Systems, in Phoenix, Arizona, USA, October 18-21, 1999, pp. 387-406.

Malaterre, P. O., Rogers, D. C., Schuurmans, J. (1998). Classification of canal control algorithms. Journal of Irrigation and Drainage Engineering, 124 (1), pp. 3-10.

Mareels, I., Weyer, E., Ooi, S. K., Cantoni, M., Li, Y., Nair, G. (August 2005). Systems engineering for irrigation systems: Successes and challenges. Annual Reviews in Control, 29 (2), pp. 191-204.

McFarlane, D. C., Glover, K. (1990). Robust controller design using normalized coprime factor plant descriptions. Lecture Notes in Control and Information Sciences. Springer-Verlag, Berlin, Germany. 
Ooi, S. K., Krutzen, M., Weyer, E. (2001). On physical and data driven modeling of irrigation channels. Control Engineering Practice, 13 (4), 461-471.

Overloop, P. J. van, Schuurmans, J., Brouwer, R., Burt, C. M. (2005). Multiplemodel optimization of proportional integral controllers on canals. Journal of Irrigation And Drainage Engineering, March/April 2005, 131 (2), pp. 190196.

Schuurmans, J. (1992). Controller design for a regional downstream controlled canal. Delft University of Technology, Laboratory for Measurement and Control, Master Thesis.

Schuurmans, J., Hof, A., Dijkstra, S., Bosgra, O. H., Brouwer, R. (1999). Simple water level controller for irrigation and drainage canals. Journal of Irrigation and Drainage Engineering, 125 (4), 189-195.

Skogestad, S., Postlethwaite, I. (1996). Multivariable Feedback Control (Analysis and Design). John Wiley and Sons, Chichester, UK.

Welz, I. G., Litrico, X., Fromion, V., Rijo, M., Malaterre, P. O. (2005). Stability and performance analysis of classical decentralised control of irrigation canals. In: Proceedings of the 16th IFAC World Congress, Prague, Czech Republic.

Weyer, E. (2001). System identification of an open water channel. Control Engineering Practice, 9 (12), 1289-1299.

Weyer, E. (July 2008). Control of irrigation channels. IEEE Transactions on Control Systems Technology, 16 (4), 664-675. 